\title{
CONSÓRCIO DE ESPÉCIES NATIVAS DA FLORESTA ESTACIONAL SEMIDECIDUAL COM MANDIOCA (Manihot sculenta Crantz) PARA RESTAURAÇÃO DE MATA CILIAR ${ }^{1}$
}

\author{
Camila Daronco², Antônio Carlos Galvão de Melo $^{3}$ e José Arimatéia Rabelo Machado ${ }^{3}$
}

\begin{abstract}
RESUMO - A consorciação entre árvores e culturas agrícolas de ciclo curto pode ser importante alternativa para promover a restauração florestal em pequenas propriedades rurais. Este estudo teve como objetivo avaliar o consórcio entre mudas de espécies arbóreas nativas e mandioca (Manihot sculenta Crantz) plantada nas entrelinhas do reflorestamento em área ripária no Oeste do Estado de São Paulo. Para tanto, utilizou-se o delineamento em blocos ao acaso, para comparar reflorestamento convencional com a floresta consorciada com a cultura agrícola. Foram avaliadas as variáveis relativas ao desenvolvimento das mudas (altura, diâmetro de copas, cobertura de copas e relação altura/diâmetro de copa), à mortalidade e ao impacto econômico do uso da mandioca. Não se observaram diferenças entre os tratamentos com relação às variáveis dendrométricas e mortalidade. O impacto econômico do tratamento consorciação foi positivo, pois os custos com a implantação do reflorestamento consorciado puderam ser, em parte, abatidos com a receita gerada pela exploração da mandioca. A receita obtida com uma safra de cultivo correspondeu a 32\% do custo total do sistema consorciado e fez que o custo total da restauração fosse diminuído em 19\%, quando comparado com o reflorestamento convencional.
\end{abstract}

Palavras-chave: Ecologia da restauração, Sistemas agroflorestais e Taungya.

\section{INTERCROPPING NATIVE TREE SPECIES OF SEASONAL SEMIDECIDUOUS FOREST WITH CASSAVA (Manihot sculenta Crantz) FOR RESTORATION OF RIPARIAN FOREST}

\begin{abstract}
Intercropping of trees and annual crops could be an important alternative for promoting forest restoration in small farms. The objective of this study was to evaluate the intercropping of cassava (Manihot sculenta Crantz) with native trees for restoration of natural forest in riparian areas in the western São Paulo State, Brazil. The experimental design was randomized blocks with six replications for comparing two restoration systems: pure afforestation and afforestation intercropped with cassava. It was evaluated the variables related to seedling development (height, crown diameter, crown cover, and height/crown diameter ratio), mortality and the economic impact were assessed for using cassava. No differences were found between treatments for any dendrometrical variable and mortality. Economic impact of the intercroppoing treatment was positive because costs for establishment of intercropped system were partially reduced by the income from the cassava crop, which covered $32 \%$ of total costs. Cassava income made final costs $19 \%$ lesser than restoration in the pure afforestation system.
\end{abstract}

Keywords: Restoration ecology, Agroforestry and Taungya.

\footnotetext{
${ }^{1}$ Recebido em 26.03.2011 e aceito para publicação em 29.03.2012

${ }^{2}$ Graduada em Ciências Biológicas pela Universidade Estadual Paulista Júlio de Mesquita Filho (2009) E-mail: <camiladaronco@yahoo.com.br>.

${ }^{3}$ Instituto Florestal do Estado de São Paulo, IF, Brasil. E-mail: <acgmelo@gmail.com>e <j.rabelomachado@yahoo.com.br>.
} 


\section{INTRODUÇÃO}

A restauração de ecossistemas ripários tem sido apontada como uma das principais medidas para a conservação da natureza, entretanto esse processo tem alto custo financeiro e exige estudos a fim de se evitarem possíveis erros que podem levar ao fracasso do plantio e a prejuízo econômico (RODRIGUES et al., 2007). A resistência do produtor rural às ações de restauração pode ser considerada natural, pois estas não fazem parte do rol das atividades para as quais ele está técnica e culturalmente preparado (CULLEN JR. et al., 2003; AGYEMAN et al., 2003). É vital, portanto, desenvolver soluções econômicas e práticas agrícolas que tornem possível ao produtor melhorar sua condição de vida, enquanto colabora com a preservação e recuperação de remanescentes florestais (RODRIGUES et al., 2007). A consorciação com culturas agrícolas, ao menos na fase de implantação da floresta, surge como uma das técnicas alternativas que podem tornar a recuperação mais atrativa financeiramente para o agricultor (DURIGAN, 1999).

A consorciação de espécies florestais com cultivos agrícolas tem como principal objetivo otimizar a produção e o retorno econômico por unidade de área em dado período de tempo (EHIAGBONARE, 2006) e leva o nome específico de taungya, sendo classificado como uma prática agroflorestal (MAC DICKEN; VERGARA, 1990; DUBOIS, 2008). Varella (2003) afirmou que a introdução de espécies semiperenes e perenes, juntamente com cultivos agrícolas, possibilita renda no médio e no longo prazo, reduz o custo com roçadas e capinas, auxilia no controle da erosão e da luminosidade, aumenta a concentração de matéria orgânica do solo e gera sustentabilidade do sistema. Para esse mesmo autor, o sistema taungya apresenta melhores resultados dos pontos de vista econômico, social e ambiental, quando comparado com o sistema tradicional de monocultivo agrícola.

Não são raros os estudos sobre a consorciação de cultivos agrícolas e cultivos florestais comerciais (aí abarcados os gêneros Pinus, Eucalyptus e Ilex), podendo ser citados Gurgel Filho (1962), Baggio et al. (1982), Schreiner (1982), Schreiner e Baggio (1984), Schreiner e Balloni (1986), Schreiner (1989), Croce e Nadal (1993), Cruz et al. (1996), Rodigheri (1997), Santos e Paiva (2002) e Souza (2007). A maior parte desses estudos indica retorno econômico significativo.
Também para reflorestamentos de restauração há relatos sobre resultados positivos com relação ao uso de diferentes formas de associação de culturas agrícolas e espécies florestais (RODRIGUES et al., 2007; BARBOSA, 2008; COUTINHO et al., 2008; RODRIGUES et al., 2008).

Em estudo desenvolvido por Rodrigues et al. (2007) com agricultores do Movimento Sem Terra (MST) no Pontal do Paranapanema que se utilizaram da consorciação floresta $\mathrm{x}$ agricultura para a recomposição da reserva legal em assentamento rural, ficou demonstrado que a produção agrícola nesse sistema tem potencial para a geração de renda e bens de consumo.

Apesar de a legislação permitir o uso do manejo florestal sustentável como estratégia para realizar a restauração ecológica (BRASIL, 2006; SÃO PAULO, 2010), os órgãos de fiscalização e licenciamento florestal nem sempre possuem informações técnico-científicas que permitam a edição de normas e rotinas e operacionalização do licenciamento de ações de restauração.

Este estudo teve como objetivo avaliar os efeitos da consorciação de Manihot sculenta Crantz sobre o crescimento e sobrevivência das espécies arbóreas e o impacto econômico da consorciação na restauração de matas ciliares.

\section{MATERIAL E MÉTODOS}

\subsection{Local do estudo}

O estudo foi instalado em zona ripária desprovida de vegetação na Fazenda Cananeia, Município de Cândido Mota, SP, localizada sob as coordenadas geográficas $22^{\circ} 32^{\prime} \mathrm{S}$ e $50^{\circ} 28^{\prime} \mathrm{W}$, em altitude de $430 \mathrm{~m}$ acima do nível do mar. O clima é de transição entre os tipos Cwa e Cfa, segundo a classificação de Köppen (PULITANO et al., 2004), e a vegetação nativa do local antes do desmatamento era Floresta Estacional Semidecidual, segundo a classificação de Veloso et al. (1991). O solo na área experimental é do tipo Latossolo Vermelho Eutroférrico típico, A moderado, textura argilosa (PULITANO et al., 2004).

A área do experimento foi ocupada por culturas agrícolas, plantadas em regime de exploração de duas safras anuais durante quase 40 anos e, antes disso, por pastagens. 


\subsection{Delineamento experimental}

Dois tratamentos foram comparados: o testemunha, que consistiu no reflorestamento com espécies nativas, em espaçamento $3 \mathrm{~m} \times 2 \mathrm{~m}$; e a consorciação, que consistiu no reflorestamento, nas mesmas condições do tratamentotestemunha, consorciado com mandioca (Manihot sculenta), que foi plantada nas entrelinhas das mudas arbóreas, com espaçamento de $50 \mathrm{~cm}$ entre as plantas de mandioca, o que implicou espaçamento de 3,0 m x 0,5 m (Figura 1).

Os tratamentos foram instalados em delineamento experimental de blocos ao acaso com seis repetições. Em junho de 2008, foram plantadas 25 mudas de árvores em cada parcela, e em novembro do mesmo ano foram plantadas 100 ramas de mandioca no tratamento consorciação.

O preparo do solo foi realizado por meio de gradagem leve, seguida do uso de riscador em profundidade de $30 \mathrm{~cm}$ nas linhas de plantio das mudas. Em vista da alta fertilidade natural do solo, não foram aplicados fertilizantes ou corretivos. A mandioca foi plantada após o uso de riscador, utilizando-se de ramas de $20 \mathrm{~cm}$ de comprimento, distantes $50 \mathrm{~cm}$ entre si.

As mudas de 37 espécies arbóreas típicas de Floresta Estacional Semidecidual (Tabela 1) foram produzidas em sacos plásticos de 1,5 L.

A altura das mudas foi medida na ocasião do plantio, aos 6, 12 e 15 meses. O diâmetro de copa foi tomado nos mesmos períodos, exceto na ocasião do plantio.

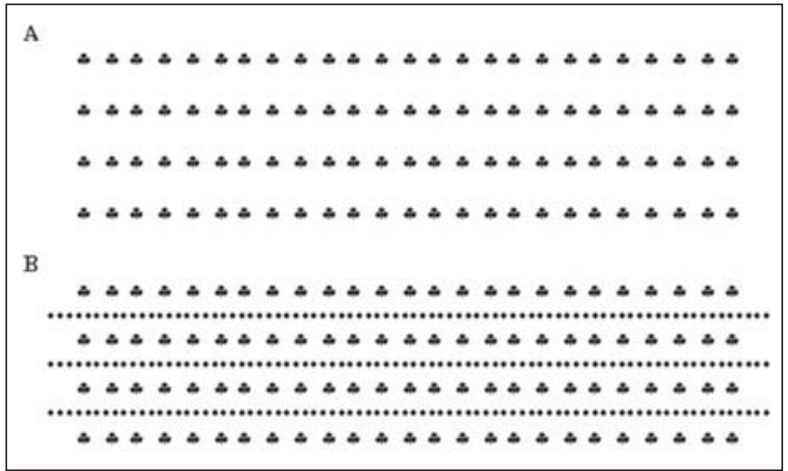

Figura 1 - A: Tratamento Testemunha; B: Tratamento consorciação (* = mudas arbóreas e $*$ plantas de mandioca) implantados em reflorestamento de restauração em Cândido Mota, SP.

Figure 1 - A: Control treatment; B: Intercropping treatment (* = trees seedlings and $*$ cassava plants) carried out in Cândido Mota, SP.

\subsection{Coleta de dados dendrométricos e informações sobre rendimento, custo e receita}

Para a medição de altura e diâmetro de copas foi utilizada régua dendrométrica telescópica. O diâmetro de copa para cada muda foi obtido pela média do maior e do menor diâmetro de cada copa. Obteve-se a cobertura de copas pela razão entre o somatório das áreas de copa individuais e a área da parcela. Na última medição, os indivíduos mortos foram registrados para análise de mortalidade. Para cada muda, aos 15 meses foi calculada a razão altura/diâmetro de copa.

Considerando que rendimentos econômicos são indicadores de sustentabilidade em SAFs (DANIEL et al., 2000), para a avaliação comparativa de custos dos dois tratamentos, todas as operações de plantio, manutenção e colheita foram acompanhadas, sendo os rendimentos operacionais registrados. A mandioca foi colhida aos 15 meses após o plantio das mudas (em setembro de 2009), sendo toda a produção pesada, em campo, em dinamômetro digital, com capacidade para 200 kg e precisão de 0,05 kg. Os valores para mão de obra, insumos e horas/máquina foram obtidos em consulta a empresas que atuam no mercado regional. O valor de venda da mandioca no mês da colheita (setembro de 2009) foi obtido em consulta ao Instituto de Economia Agrícola (2009).

Foi calculado o lucro líquido pela diferença entre receita total da colheita e custos de plantio, manutenção e colheita; e a relação benefício/custo, pela razão entre a receita total da colheita e os custos totais.

\subsection{Análise dos dados}

Para comparação das porcentagens de mortalidade e de cobertura de copas, os valores calculados foram transformados em arcosseno $\sqrt{p}$, em que p é a porcentagem da cobertura. A normalidade dos dados foi checada por meio do teste de Shapiro-Wilk. Quando os dados apresentavam os pressupostos da normalidade, a comparação entre os tratamentos foi feita por meio da ANOVA, e quando tal não acontecia recorreu-se ao teste de Kruskal-Wallis. Em ambos os casos, considerou-se o nível de significância $\alpha=5 \%$.

\section{RESULTADOS}

Até os 15 meses de idade - ocasião da colheita da mandioca - não houve diferença entre os tratamentos para nenhuma variável analisada (Tabela 2).

Revista Árvore, Viçosa-MG, v.36, n.2, p.291-299, 2012 
Tabela 1 - Espécies arbóreas de Floresta Estacional Semidecidual utilizadas para restauração de mata ciliar em Cândido Mota, SP. Table 1 - Tree species of the Seasonal Semideciduous Forest used for restoration of riparian forest in Cândido Mota, SP, Brazil.

\begin{tabular}{|c|c|c|}
\hline Nome científico & Nome popular & Família \\
\hline Allophyllus edulis (St. Hil.) Radlk & Chal chal & Sapindaceae \\
\hline Anadenanthera colubrina (Vell.) Brenan & Angico-branco & Fabaceae \\
\hline Anadenanthera macrocarpa (Benth.) Brenan & Angico & Fabaceae \\
\hline Aspidosperma cylindrocarpon Müll. Arg. & Peroba-poca & Apocynaceae \\
\hline Astronium graveolens Jacq. & Guaritá & Anacardiaceae \\
\hline Balfourodendron riedelianum (Engl.) Engl. & Pau-marfim & Rutaceae \\
\hline Bastardiopsis densiflora (Hook. \& Arn.) Hassl. & Louro-branco & Malvaceae \\
\hline Calycophyllum multiflorum Griseb. & Pau-branco & Rubiaceae \\
\hline Cariniana estrellensis (Raddi) Kuntze & Jequitibá-branco & Lecythidaceae \\
\hline Ceiba speciosa (A.St.-Hil.) Ravenna & Paineira & Malvaceae \\
\hline Cordia myxa L. & Baba-de-boi & Boraginaceae \\
\hline Croton urucurana Baill. & Sangra-d'água & Euphorbiaceae \\
\hline Cytharexylum myrianthum Cham. & Pau-viola & Verbenaceae \\
\hline Endlicheria paniculata (Spreng.) J. F. Macbr. & Canela-frade & Lauraceae \\
\hline Enterolobium contortisiliquum (Vell.) Morong & Timburi & Fabaceae \\
\hline Esenbeckia leiocarpa Engl. & Guarantã & Rutaceae \\
\hline Gochnatia polymorpha (Less.) Cabrera & Candeia & Asteraceae \\
\hline Handroanthus heptaphyllus (Vell.) Mattos & Ipê-roxo & Bignoniaceae \\
\hline Handroanthus impetiginosus (Mart. ex DC.) Mattos & Ipê-roxo-de-bola & Bignoniaceae \\
\hline Heliocarpus americanus $\mathrm{L}$. & Algodoeiro & Malvaceae \\
\hline Hymenaea courbaril L. & Jatobá & Fabaceae \\
\hline Inga laurina (Sw.) Willd & Ingá-branco & Fabaceae \\
\hline Inga uruguensis Hook. \& Arn. & Ingá-brejo & Fabaceae \\
\hline Lacistema hasslerianum Chodat & - & Lacistemataceae \\
\hline Maclura tinctoria (L.) D. Don ex Steud. & Taiuva & Moraceae \\
\hline Maytenus aquifolium Chodat & Espinheira-santa & Celastraceae \\
\hline Mollinedia widgrenii A.DC. & - & Monimiaceae \\
\hline Myrocarpus frondosus Allemão & Cabreúva-parda & Fabaceae \\
\hline Myroxylon peruiferum L.f. & Cabreúva-vermelha & Fabaceae \\
\hline Nectandra cuspidata Nees \& Mart & Canelão & Lauraceae \\
\hline Parapiptadenia rigida (Benth.) Brean & Angico-vermelho & Fabaceae \\
\hline Peltophorum dubium (Spreng.) Taub. & Canafístula & Fabaceae \\
\hline Pouteria ramiflora (Mart.) Radlk. & Leiteiro-preto & Sapotaceae \\
\hline Savia dictyocarpa Müll. Arg. & Guaraiuva & Phyllanthaceae \\
\hline Terminalia argentea Mart. & Capitão & Combretaceae \\
\hline Trichilia pallida $\mathrm{Sw}$. & Baga-de-morcego & Meliaceae \\
\hline Zeyheria tuberculosa (Vell.) Bureau & Ipê-tabaco & Bignoniaceae \\
\hline
\end{tabular}

Revista Árvore, Viçosa-MG, v.36, n.2, p.291-299, 2012 
Tabela 2 - Altura, diâmetro de copa, cobertura de copas, mortalidade e relação altura/diâmetro de copa em plantio convencional ou em consórcio com mandioca (Manihot sculenta Crantz) em Cândido Mota, SP (média \pm desvio-padrão).

Table 2 - Height, canopy diameter, canopy cover, mortality and height/canopy diameter ratio for a conventional planting or intercropped with cassava (Manihot sculenta Crantz) in Cândido Mota, SP, Brazil (mean \pm standard deviation).

\begin{tabular}{lccc}
\hline \multicolumn{1}{c}{ Variáveis } & Consórcio & Reflorestamento convencional & Análise de variância \\
\hline Altura inicial $(\mathrm{m})$ & $0,48 \pm 0,03$ & $0,46 \pm 0,04$ & $\mathrm{~F}=0,537 ; \mathrm{p}=0,514$ \\
Altura aos seis meses $(\mathrm{m})$ & $0,91 \pm 0,21$ & $0,86 \pm 0,16$ & $\mathrm{~F}=0,205 ; \mathrm{p}=0,663$ \\
Altura aos 12 meses $(\mathrm{m})$ & $1,43 \pm 0,34$ & $1,28 \pm 0,25$ & $\mathrm{~F}=0,679 ; \mathrm{p}=0,566$ \\
Altura aos 15 meses $(\mathrm{m})$ & $1,79 \pm 0,49$ & $1,70 \pm 0,40$ & $\mathrm{~F}=0,122 ; \mathrm{p}=0,732$ \\
Diâmetro de copa aos seis meses $(\mathrm{m})$ & $0,68 \pm 0,21$ & $0,65 \pm 0,16$ & $\mathrm{~F}=0,040 ; \mathrm{p}=0,839$ \\
Diâmetro de copa aos 12 meses $(\mathrm{m})$ & $1,07 \pm 0,36$ & $1,06 \pm 0,31$ & $\mathrm{~F}=0,001 ; \mathrm{p}=0,970$ \\
Diâmetro de copa aos 15 meses $(\mathrm{m})$ & $1,42 \pm 0,42$ & $1,36 \pm 0,37$ & $\mathrm{~F}=0,082 ; \mathrm{p}=0,777$ \\
Cobertura de copas $(\%)$ & $45,31 \pm 21,40$ & $44,56 \pm 20,00$ & $\mathrm{H}=0,231 ; \mathrm{p}=0,631$ \\
Mortalidade $(\%)$ & $10,0 \pm 11,0$ & $14,7 \pm 13,5$ & $\mathrm{~F}=0,449 ; \mathrm{p}=0,524$ \\
Relação altura $:$ diâmetro de copa & $1,27 \pm 0,12$ & $\mathrm{H}=0,161 ; \mathrm{p}=0,689$ & \\
\hline
\end{tabular}

Os custos de implantação e manutenção no sistema consorciado foram de R \$8.161,21 (Tabela 3), e no sistema de plantio exclusivo com mudas de espécies arbóreas os custos foram de R\$6.918,61 (Tabela 4). Com relação aos custos, o que diferencia os dois tratamentos são as operações necessárias ao plantio e à colheita da mandioca, que foi realizada apenas no tratamento consorciação e totalizou R $\$ 1.242,60$ por hectare.

O cultivo da mandioca no sistema consorciado apresentou produtividade média de $6.113,3 \mathrm{~kg} \cdot \mathrm{ha}^{-1}$. Considerando um valor de mercado cotado em $\mathrm{R} \$ 0,42$ por quilograma na época da colheita (INSTITUTO DE ECONOMIA AGRÍCOLA, 2009), obteve-se a receita de $\mathrm{R} \$ 2.567,60$ por hectare. Tal receita seria suficiente para abater $32 \%$ do custo total no tratamento consorciação. O resultado financeiro permite afirmar que o hectare de floresta restaurada, utilizando-se a consorciação, teria um custo final de $\mathrm{R} \$ 5.593,61$, que é $19 \%$ menor que o custo de restauração em sistema de plantio convencional com mudas de espécies arbóreas, sem consorciação (Tabela 4). O rendimento da colheita em 1 ha foi de 550,75 kg de mandioca por homem/dia.

Para o plantio da mandioca, o lucro obtido foi de $\mathrm{R} \$ 1.327,00$ e a relação Benefício/Custo, 2,07, mostrando a viabilidade econômica da consorciação para as condições apresentadas.

\section{DISCUSSÃO}

A ausência de diferença entre os tratamentos com relação às variáveis analisadas indica que a utilização da mandioca em consórcio não trouxe prejuízos ao desenvolvimento das árvores. Embora houvesse a possibilidade de a consorciação induzir a competição por água, nutrientes e luz, a depender da densidade das plantas introduzidas no sistema (DE PAULA; DE PAULA, 2003), a ausência de diferenças da relação altura/diâmetro de copa entre tratamentos indica que não ocorreu estiolamento das árvores, sugerindo que a lotação de plantas de mandioca utilizada não chegou a promover competição entre mudas arbóreas e a cultura agrícola. Tal resultado é corroborado pela inexistência de diferença nas taxas de mortalidade entre os tratamentos estudados (Tabela 2).

Em outros estudos sobre consorciação em reflorestamentos com uma única espécie também não foram observadas diferenças significativas aos 15 meses de idade entre cultivo solteiro ou consorciado, com relação à sobrevivência das árvores (BAGGIO et al., 1982; CRUZ et al., 1996; SCHREINER; BAGGIO, 1984; SCHREINER; BALLONI, 1986; PASSOS, 1990). Para altura, como neste estudo, não foi observada diferença entre tratamentos consorciados e convencionais nos estudos que trataram de reflorestamentos puros desenvolvidos por Baggio et al. (1982), Cruz et al. (1996), Passos (1990), Schreiner e Balloni (1986) e Schreiner (1989). Também não foi observada diferença significativa para cobertura de copas. Os valores médios de cobertura de copas encontrados no tratamento consorciação (45,31\%) e testemunha (44,56\%) aos 15 meses foram muito próximos aos encontrados por Melo et al. (2007) em reflorestamento heterogêneo aos 13 meses (44,67\%), na região do Médio Paranapanema.

Revista Árvore, Viçosa-MG, v.36, n.2, p.291-299, 2012 
Tabela 3 - Custos para implantação e manutenção, por hectare, até os 15 meses de idade, de reflorestamento com espécies arbóreas nativas consorciadas com mandioca em Cândido Mota, SP.

Table 3 - Costs for installation and maintenance, per hectare, until 15 months of age, in reforestation with native tree species intercropped with cassava in Cândido Mota, SP, Brazil.

\begin{tabular}{llclrr}
\hline \multicolumn{1}{c}{ Atividade } & \multicolumn{1}{c}{ Item } & Quantidade & Unidade & Valor unitário (R\$) & Valor total (R\$) \\
\hline Preparo solo & Trator + grade niveladora & 2,78 & Hora.máquina & 110,00 & 305,80 \\
& Trator + riscador & 1,39 & Hora.máquina & 110,00 & 152,90 \\
& Tratorista & 0,55 & Homem.dia & 40,00 & 22,00 \\
\hline Plantio & Transporte mudas & 60 & Quilômetro & 1,50 & 90,00 \\
& Mão de obra & 10,55 & Homem.dia & 33,00 & 348,15 \\
& Mudas & 1667 & Unidades & 1,00 & $1.667,00$ \\
& Trator + carreta & 16,65 & Hora.máquina & 110,00 & $1.831,50$ \\
\hline Plantio mandioca & Mão de obra & 11,1 & Homem.dia & 33,00 & 366,30 \\
& Ramas & 4,1 & m & 40,00 & 164,00 \\
\hline Replantio & Transporte mudas & 60 & Quilômetro & 1,50 & 90,00 \\
& Mão de obra & 4,44 & Homem.dia & 33,00 & 146,52 \\
& Mudas & 311 & Unidades & 1,00 & 311,00 \\
& Trator + carreta & 8,88 & Hora.máquina & 110,00 & 976,80 \\
\hline Capinas (4x) & Mão de obra & 8,88 & Homem.dia & 33,00 & 293,04 \\
\hline Controle de formigas $(4 x)$ & Mão de obra & 5,0 & Homem.dia & 33,00 & 165,00 \\
& Formicida isca & 61,05 & kg & 8,50 & 518,90 \\
\hline Colheita mandioca & Mão de obra & 11,1 & Homem.dia & 33,00 & 366,3 \\
& Trator & 3,00 & Hora.máquina & 110,00 & 330,00 \\
& Tratorista & 0,40 & Homem.dia & 40,00 & 16,00 \\
\hline Total & & & & $8.161,21$ \\
\hline
\end{tabular}

Tabela 4 - Custos para implantação e manutenção, por hectare, até os 15 meses de idade, de reflorestamento com espécies arbóreas nativas em Cândido Mota, SP.

Table 4 - Costs for installation and maintenance, per hectare, until 15 months of age, in reforestation with native tree species in Cândido Mota, SP, Brazil.

\begin{tabular}{llclrr}
\hline Atividade & \multicolumn{1}{c}{ Item } & Quantidade & Unidade & Valor unitário (R\$) & Valor total (R\$) \\
\hline Preparo solo & Trator + grade niveladora & 2,78 & Hora.máquina & 110,00 & 305,80 \\
& Trator + riscador & 1,39 & Hora.máquina & 110,00 & 152,90 \\
& Tratorista & 0,55 & Homem.dia & 40,00 & 22,00 \\
\hline Plantio & Transporte mudas & 60 & Quilômetro & 1,50 & 90,00 \\
& Mão de obra & 10,55 & Homem.dia & 33,00 & 348,15 \\
& Mudas & 1667 & Unidades & 1,00 & $1.667,00$ \\
& Trator + carreta & 16,65 & Hora.máquina & 110,00 & $1.831,50$ \\
\hline Replantio & Transporte mudas & 60 & Quilômetro & 1,50 & 90,00 \\
& Mão de obra & 4,44 & Homem.dia & 33,00 & 146,52 \\
& Mudas & 311 & Unidades & 1,00 & 311,00 \\
& Trator + carreta & 8,88 & Hora.máquina & 110,00 & 976,80 \\
\hline Capinas (4x) & Mão de obra & 8,88 & Homem.dia & 33,00 & 293,04 \\
\hline Controle de formigas (4x) & Mão de obra & 5,0 & Homem.dia & 33,00 & 165,00 \\
& Formicida isca & 61,05 & kg & 8,50 & 518,90 \\
\hline Total & & & & & $6.918,61$ \\
\hline
\end{tabular}

Revista Árvore, Viçosa-MG, v.36, n.2, p.291-299, 2012 
Os custos com o reflorestamento foram, em parte, abatidos pelo cultivo intercalar de mandioca. Entretanto, a produtividade média da mandioca registrada neste estudo foi cerca de três vezes menor que a média regional (INSTITUTO DE ECONOMIA AGRÍCOLA, 2009) que, no biênio 2008-2009, foi de $19.043,0$ kg.ha-1 ${ }^{-}$Tal diferença é explicada pela lotação de plantas de mandioca que, quando em plantio solteiro, é espaçada em 1,0 m x 0,5 m. Portanto, pode-se obter maior produtividade no sistema consorciado caso se aumente o número de plantas de mandioca, porém são necessários estudos sobre os efeitos desse aumento de densidade no desenvolvimento das mudas arbóreas.

O preço da mandioca, entretanto, pode apresentar grande oscilação de um ano para o outro. No mesmo período de coleta (setembro), o preço do quilograma variou de $\mathrm{R} \$ 0,18$ a R \$0,57 nos anos de 2006 e 2008, respectivamente (INSTITUTO DE ECONOMIA AGRÍCOLA, 2009). Esse comportamento dos preços é um importante alerta que deve ser levado em conta na hora do planejamento do plantio. Tal flutuação pode implicar aumento dos custos da restauração ao invés de se conseguir sua redução.

Furlaneto et al. (2007) afirmaram que o rendimento médio da colheita manual da mandioca varia de $700 \mathrm{~kg}$ a $1.500 \mathrm{~kg}$ por homem/dia, ou seja, superior ao encontrado neste estudo (550,75 kg). Assim, os resultados aqui apresentados podem ser ainda melhores caso se consigam rendimentos operacionais próximos aos encontrados na literatura para o cultivo convencional de mandioca.

Este estudo ratifica outros realizados com diferentes desenhos de consorciação entre árvores e cultura agrícola (GURGEL FILHO, 1962; BAGGIO, 1982; SCHREINER; BAGGIO, 1984; SCHREINER; BALLONI, 1986; RODIGHERI, 1997), segundo os quais o plantio de culturas agrícolas em consórcio com espécies arbóreas permite que os gastos do reflorestamento sejam, em parte, ressarcidos pela comercialização dos produtos.

\section{CONCLUSÕES}

O crescimento das espécies arbóreas não foi afetado pelo cultivo intercalar de mandioca.
O impacto econômico da consorciação foi positivo. A receita gerada pela venda da mandioca abateu não só os custos dessa cultura, como também parte do custo do reflorestamento.

\section{AGRADECIMENTOS}

À Fundação de Amparo à Pesquisa de São Paulo - FAPESP, pela bolsa de iniciação científica concedida ao primeiro autor; ao Instituto Florestal - IF, pelo financiamento parcial do projeto; à Dra. Giselda Durigan pelas sugestões e apoio à implantação do experimento; e à Fazenda Cananeia, por disponibilizar área e mão de obra para a realização do experimento.

\section{REFERÊNCIAS}

AGYEMAN, V. K. et al. Revising the taungya plantation system: new revenue-sharing proposals from Ghana. Unasylva, v.54, n.1, p.40-43, 2003.

BAGGIO, A. J et al. Consorciação das culturas da erva-mate (Illex paraguariensis A. St. Hilaire) e feijão (Phaseolus vulgaris, L.) no Paraná.

Boletim de Pesquisa Florestal, v.2, n.4, p.75-90, 1982.

BARBOSA, E. N. Fetaesp na implantação de sistemas agroflorestais - SAFs. In:

Recuperação florestal: um olhar social. São Paulo: Secretaria do Meio Ambiente, 2008. p.105-107.

BRASIL. Resolução Conselho Nacional do Meio Ambiente $n^{\circ}$ 369, de 28 de março de 2006.

Diário Oficial da União, n. 61. Brasília, de 29 de março de 2006. Seção 1. p.150-151.

COUTINHO, M. P et al. Oportunidades para recuperação de áreas degradadas na Bacia Hidrográfica do São Francisco. In: FELFILI, J. M.; SAMPAIO, J. C.; CORREIA, C. R. M. A. Bases para recuperação de áreas degradadas na Bacia do São Francisco. Brasília: Centro de Referência em Conservação da Natureza e Recuperação de Áreas Degradadas (CRAD), 2008. p.41-44.

CRUZ, S. F. et al. Consorciação entre espécies de Pinus e culturas agrícolas. Revista do Instituto Florestal, v.8, n.2, p.135-144, 1996.

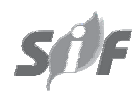

Revista Árvore, Viçosa-MG, v.36, n.2, p.291-299, 2012 
CULLEN JÚNIOR, L. et al. Trampolins ecológicos e zonas de benefício múltiplo: ferramentas agroflorestais para a conservação de paisagens rurais fragmentadas na Floresta Atlântica brasileira. Natureza \& Conservação, v.1, n.1, p.37-46, 2003.

CROCE, D. M.; NADAL, R. Viabilidade técnicoeconômica de sistemas de produção de erva-mate (Illex paraguariensis A. St. Hill.) consorciado com culturas anuais. In: CONGRESSO FLORESTAL PANAMERICANO, 1.,/CONGRESSO FLORESTAL BRASILEIRO, 7., 1993, Curitiba. Anais... São Paulo: Sociedade Brasileira de Engenheiros Florestais/Sociedade Brasileira de Silvicultura, 1993. p.403-406.

DANIEL, O. et al. Proposta de um conjunto mínimo de indicadores sócio-econômicos para o monitoramento da sustentabilidade em sistemas agroflorestais. Revista Árvore, v.24, n.3, p.283-290, 2000.

DE PAULA, R. C.; DE PAULA, N. F. Sistemas agroflorestais. In: VALERI, S. V.; POLITANO, W. (Eds.). Manejo e recuperação florestal. Jaboticabal: Funep, 2003. 180p.

DUBOIS, J. Classificação e breve caracterização de SAFs e práticas agroflorestais . In: MAY, P. H.; TROVATTO, C. M. M. (Coord.). Manual agroflorestal para a Mata Atlântica. Brasília: Ministério do Desenvolvimento Agrário, Secretaria da Agricultura Familiar, 2008. p.15-62.

DURIGAN, G. Técnicas silviculturais aplicadas à restauração de ecossistemas. In: SIMPÓSIO SOBRE RESTAURAÇÃO ECOLÓGICADE ECOSSISTEMAS NATURAIS, 1., 1999, Piracicaba. Anais... Piracicaba: ESALQ/USP, 1999.

EHIAGBONARE, J. E. Effect of taungya on regeneration of endemic forest tree species in Nigeria: Edo State Nigeria as a case study.

African Journal of Biotechnology, v.5, n.18, p.1608-1611, 2006.

FURLANETO, P. B.; KANTHACK, R. A. D.; ESPERANCINI, M. S. T. Análise econômica da cultura da mandioca no Médio Paranapanema, Estado de São Paulo. Informações

Econômicas, v.37, n.10, p. 20-26, 2007.
GURGEL FILHO, O. A. Plantio de eucalipto consorciado com milho. Silvicultura em São Paulo, v.1, p.85-102, 1962.

INSTITUTO DE ECONOMIAAGRÍCOLA - IEA. Disponível em: <http://ciagri.iea.sp.gov.br/ bancoiea/Precos_Medios.aspx?cod_sis=2>. Acesso em: 30 de dez. de 2009.

MAC DICKEN, K. G.; VERGARA, N. T. Introduction to agroforestry. In: MAC DICKEN, K. G.; VERGARA, N. T. Agroforestry: classification and management. New York: John Wiley \& Sons, 1990. p.1-30.

MELO, A. C. G.; MIRANDA, D. L. C.; DURIGAN, G. Cobertura de copas como indicador de desenvolvimento estrutural de reflorestamentos de restauração de matas ciliares no Médio Vale do Paranapanema, SP, Brasil. Revista Árvore, v.31, n.2, p.321-328, 2007.

PASSOS, C. A. M. Comportamento inicial do eucalipto (Eucalyptus grandis W. Hill ex. Maiden) em plantio consorciado com feijão (Phaseolus vulgaris L.), no Vale do Rio Doce, Minas Gerais. 1990. 64f. Disertação (Mestrado em Ciência Florestal) Universidade Federal de Viçosa, Viçosa, MG, 1990.

PULiTANO, F. M.; DURIGAN, G.; DiAS, L. E. A mata ciliar da Fazenda Cananéia: estrutura e composição florística em dois setores com idades diferentes. In: BÔAS, O. V.; DURIGAN, G.

Pesquisa em conservação e recuperação ambiental no oeste paulista: resultados da cooperação Brasil/Japão. São Paulo: Páginas \& Letras, 2004. p.419-445.

RODIGHERI, H. R. Rentabilidade econômica comparativa entre plantios florestais e sistemas agroflorestais com erva-mate, eucalipto e pinus e as culturas do feijão, milho, soja e trigo. Colombo: Embrapa - CNPF, 1997. 36p. (Circular Técnica, 26).

RODRIGUES, E. R. et al. Avaliação econômica de sistemas agroflorestais implantados para a recuperação de Reserva Legal no Pontal do Paranapanema, São Paulo. Revista Árvore, v.31, p.941-948, 2007. 
RODRIGUES, E. R. et al. O uso do sistema agroflorestal taungya na restauração de Reservas Legais: indicadores econômicos. Revista Floresta, v.38, n.3, p.517-525, 2008.

SANTOS, M. J. C.; PAIVA, S. N. Os sistemas agroflorestais como alternativa econômica em pequenas propriedades rurais: estudo de caso. Ciência Florestal, v.12, n.1, p.135-141, 2002.

SÃO PAULO. Decreto 55.947 de 24 de junho de 2010. Regulamenta a Lei $n^{\circ} 13.798$, de 9 de novembro de 2009, que dispõe sobre a Política Estadual de Mudanças Climáticas. Diário Oficial do Estado de São Paulo, v.120, n.119. São Paulo, 25 de junho de 2010.Seção i, p.1-2.

SCHREINER, H. G. Consórcio das culturas de erva mate e milho. In: CONGRESSO NACIONAL SOBRE ESSÊNCIAS NATIVAS, 1982, Campos do Jordão. Anais... São Paulo: Silvicultura em São Paulo, 1982. p.812-813.

SCHREINER, H. G. Culturas intercalares de soja em reflorestamento de eucaliptos no Sul - Sudeste do Brasil. Boletim de Pesquisa Florestal, n.18/19, p.1-10, 1989.
SCHREINER, H. G.; BAGGIO, A. J. Culturas intercalares de milho (Zea mays, L.) em reflorestamento de Pinus taeda L. no sul do Paraná. Boletim de Pesquisa Florestal, n.8/9, p.26-49, 1984.

SCHREINER, H. G.; BALLONI, E. A. Consórcio das culturas de feijão (Phaseolus vulgaris, L.) e eucalipto (Eucalyptus grandis, W. Hill ex - Maiden) no Sudeste do Brasil. Boletim de Pesquisa Florestal, n.12, p.83-104, 1986.

SOUZA, A. N. et al. Viabilidade econômica de um sistema agroflorestal. Cerne, v.13, n.1, p.96-106, 2007.

VARELLA, L. B. Sustentabilidade e prospectiva do sistema taungya em comparação com a roça tradicional na Zona Bragantina do Estado do Pará. Movendo Idéias, v.8, n.14, p.73-85, 2003.

VELOSO, H. P.; RANGEL FILHO, A. L.; LIMA, J. C. A. Classificação da vegetação brasileira adaptada a um sistema universal. Rio de Janeiro: IBGE, 1991. 
